ARTICLE

Received 3 Jun 2014 | Accepted 15 Dec 2014 | Published 22 Jan $2015 \quad$ DOl: 10.1038/ncomms7123

\title{
Proteasome assembly from 155 precursors involves major conformational changes and recycling of the Pba1-Pba2 chaperone
}

\author{
Malte Kock ${ }^{1, \star}$, Maria M. Nunes ${ }^{2, \star}$, Matthias Hemann${ }^{1}$, Sebastian Kube${ }^{1}$, R. Jürgen Dohmen²,
} Franz Herzog ${ }^{1}$, Paula C. Ramos ${ }^{2} \&$ Petra Wendler ${ }^{1}$

The chaperones Ump1 and Pba1-Pba2 promote efficient biogenesis of 20S proteasome core particles from its subunits via 15S intermediates containing alpha and beta subunits, except beta7. Here we elucidate the structural role of these chaperones in late steps of core particle biogenesis using biochemical, electron microscopy, cross-linking and mass spectrometry analyses. In $15 \mathrm{~S}$ precursor complexes, Ump1 is largely unstructured, lining the inner cavity of the complex along the interface between alpha and beta subunits. The alpha and beta subunits form loosely packed rings with a wider alpha ring opening than in the 205 core particle, allowing for the Pba1-Pba2 heterodimer to be partially embedded in the central alpha ring cavity. During biogenesis, the heterodimer is expelled from the alpha ring by a restructuring event that organizes the beta ring and leads to tightening of the alpha ring opening. In this way, the Pba1-Pba2 chaperone is recycled for a new round of proteasome assembly.

\footnotetext{
${ }^{1}$ Gene Center Munich, Ludwig-Maximilians-Universität München, Feodor-Lynen Strasse 25, D-81377 Munich, Germany. ${ }^{2}$ Institute for Genetics, University of Cologne, Zülpicher Strasse 47a, D-50674 Cologne, Germany. * These authors contributed equally to this work. Correspondence and requests for materials should be addressed to P.C.R. (email: pramos@uni-koeln.de) or to P.W. (email: wendler@genzentrum.Imu.de)
} 
T he $26 \mathrm{~S}$ proteasome is a $2.5-\mathrm{MDa}$ protein complex tasked with timely degradation of polyubiquitylated proteins in eukaryotic cells. Proper function of the proteasome is vital to all eukaryotic life and malfunction has been implicated in a wide array of human diseases, including cancer and neurodegenerative disorders ${ }^{1,2}$. The $26 \mathrm{~S}$ proteasome is composed of a barrelshaped 20S core particle (CP) to the ends of which one or two 19S regulatory particles (RPs) are attached. While a high-resolution crystal structure of the eukaryotic 20S CP is known since 1997 (ref. 3), subnanometre structures of the 19S RP have only recently been determined by cryoelectron microscopy ${ }^{4-6}$.

In eukaryotes, the $20 \mathrm{~S} \mathrm{CP}$ consists of seven distinctive alpha and seven distinct beta subunits, which are assembled into stacked rings, resulting in a characteristic $\alpha 7 / \beta 7 / \beta 7 / \alpha 7$ architecture $^{3}$. The $\beta$ subunits form the proteolytic chamber, while the $\alpha$ subunits interact with regulatory complexes and promote gating of the $20 \mathrm{SCP}^{7}$. Proteasome biogenesis is an ordered process aided by several chaperones ${ }^{8}$, which are thought to promote productive and prevent promiscuous subunit interactions ${ }^{9-12}$. Early steps in the assembly of the $20 \mathrm{~S}$ proteasome are supported by the heterodimeric chaperones $\mathrm{Pba} 1-\mathrm{Pba} 2$ and $\mathrm{Pba} 3-\mathrm{Pba} 4$ (refs 13,14), which promote correct assembly of the alpha ring. Formation of an intermediate containing a complete alpha ring and the $\beta 2, \beta 3$ and $\beta 4$ subunits $^{15,16}$ requires displacement of $\mathrm{Pba} 3-\mathrm{Pba} 4$ (ref. 17). In yeast, the next detectable intermediate in the biogenesis pathway additionally contains the $17-\mathrm{kDa}$ chaperone Ump1, $\beta 5, \beta 6$ and $\beta 1$ (refs 16,18) and it is termed the $15 \mathrm{~S}$ precursor complex (PC) based on its sedimentation properties in sucrose gradients ${ }^{19}$. Addition of the $\beta 7$ subunits completes the assembly and leads to rapid dimerization of two half-proteasomes through intercalation of the C-terminal $\beta 7$ extensions between $\beta 1$ and $\beta 2$ in the opposite beta ring ${ }^{16,20}$. Once the early $20 S$ proteasome precursor is formed, autocatalytic propeptide cleavage of the proteolytic subunits $\beta 1, \beta 2$ and $\beta 5$ takes place $^{21}$. Upon activation of the proteolytic sites, the pro-peptides of $\beta 6$ and $\beta 7$ are processed ${ }^{22}$, and Ump1 is degraded as the first substrate of the nascent proteasome ${ }^{19}$. Work in mammalian cells suggested that subsequently also the Pba1-Pba2 chaperone is degraded by newly formed active $\mathrm{CP}^{23}$.

Although Pba1 and Pba2 (also known as Poc1 and Poc2) are not essential for viability in Saccharomyces cerevisiae ${ }^{13,16}$, knockout or knockdown, respectively, of the mammalian orthologue of Pbal, termed PAC1, causes embryonic lethality in mice or severe defects in proteasome assembly in cultured human cells ${ }^{23,24}$. The two proteins form a stable heterodimer, which was reconstituted and crystallized with mature $20 \mathrm{~S} C \mathrm{CP}$ from yeast ${ }^{25}$. In this structure, the C-terminal HbYX motif of Pbal interacts with the intersubunit pocket between $\alpha 5$ and $\alpha 6$, which can also bind to the HbYX motif of Blm10 or of the 19S RP subunit Rpt5 (refs 7,26,27). Despite featuring a similar HbYX motif, $\mathrm{Pba} 2$ interacts in a different manner with the $\alpha 6 / \alpha 7$ pocket making a smaller contribution to binding of the heterodimer ${ }^{25,28}$. The central channel, which is formed in the alpha ring of activated $\mathrm{CP}^{29,30}$, is closed in Pba1-Pba2-bound proteasomes ${ }^{25}$.

Ump1 was the first chaperone found to be involved in proteasome biogenesis. Its deletion is not lethal in yeast, but makes cells hypersensitive to various forms of stress and leads to an accumulation of aberrant $20 \mathrm{~S}$ CPs with unprocessed beta subunits ${ }^{19}$. The deletion of Umpl can rescue lethality caused by deletions of the pro-peptides of either $\beta 5$ or $\beta 6$, pointing to a possible physical interaction between Ump1 and these pro-peptides ${ }^{19,16}$.

Structural analyses have been reported for precursors of prokaryotic proteasomes, which assemble without dedicated chaperones $^{31,32}$. No structures are available for precursors of the more complex eukaryotic $\mathrm{CP}$ bearing the above-mentioned chaperones Ump1 and Pba1-Pba2.
Here we use single-particle electron microscopy (EM) combined with chemical cross-linking and mass spectrometric (CX-MS) analysis to investigate the subunit arrangement and chaperone localization in proteasomal precursors. Compared with the structure of a Pbal-Pba2-20S complex, we find substantial conformational changes within the alpha and beta rings of $15 \mathrm{~S}$ PCs, with the Pba1-Pba2 heterodimer semiembedded in the centre of the alpha ring. Upon maturation of the 20S CP, the Pba1-Pba2 chaperone is ejected from the pore and recycled. Tracing the amino-acid chain of Ump1 in the $15 \mathrm{~S}$ PC reveals interactions with multiple proteasomal alpha and beta subunits indicating an unstructured, extended conformation of this chaperone.

\section{Results}

Structure of the $15 \mathrm{~S}$ proteasome PC. The abundance of the $15 \mathrm{~S}$ intermediate in the proteasome biogenesis pathway is very low in wild-type cells. In order to enrich the cellular $15 \mathrm{~S}$ precursor population and to enable selective affinity purification, we used a yeast strain lacking the $C$-terminal extension of $\beta 7(\beta 7 \Delta C T E)^{18}$ and expressing an N-terminally FLAG- $6 \times$ His $(\mathrm{FH})$-tagged version of Ump1. The $\beta 7 \Delta$ CTE mutation does not affect the structure of the $15 \mathrm{~S}$ intermediate because the $\beta 7$ subunit is absent from this complex ${ }^{18}$; however, lack of the CTE causes an accumulation of the 15S PC because it inhibits 20S CP formation by complex dimerization ${ }^{20}$.

We used a tagged version of Ump1 for a selective purification of the 15S PC because this assembly chaperone represents the only polypeptide that is associated with the 15S PC but not with $20 \mathrm{~S}$ CPs. Initial affinity purifications of FH-tagged complexes from this strain not only yielded the 15S PC, but also many complexes lacking the $\mathrm{Pba} 1-\mathrm{Pba} 2$ chaperone, some of which were associated instead with Blm10 (Fig. 1a). To increase homogeneity of the precursor population, we introduced a deletion of the $B L M 10$ gene and overexpressed the Pba1-Pba2 heterodimer. A strain with these modifications produced the $15 \mathrm{~S}$ PC as the dominant proteasomal complex (Fig. 1a,b). The subunits of the purified PCs derived from this strain were separated by SDSPAGE and subsequent peptide mass fingerprint analysis detected Pba1, Pba2, Ump1 and all 20S subunits but $\beta 7$ (Fig. 1c). The identification of precursor forms of $\beta 2$ and $\beta 5$ in this preparation confirmed that the purified ${ }^{\mathrm{FH}} 15 \mathrm{~S}$ complexes represent assembly intermediates (Supplementary Fig. 1a). These complexes were analyzed by negative stain EM and single-particle imageprocessing (Fig. 1d, Supplementary Fig. 1b). Multivariate statistical analysis of top view images did not reveal any heterogeneity caused by partial dissociation of Pba1-Pba2 in this data set (Supplementary Fig. 1c). Analysis of reference-free twodimensional (2D) class averages revealed a sevenfold rotational symmetry in the top views of the complex and two-layered side views (Fig. 1d). The asymmetric three-dimensional (3D) reconstruction of the $\mathrm{FH}_{15 \mathrm{~S}}$ complex has a diameter of $128 \AA$ and a height of $112 \AA$ (Fig. 1e). It shows a double ring structure with an internal diameter of $\sim 30 \AA$, which is capped by a dimeric density on one side. The Pba1-Pba2 heterodimer and the alpha subunits fit the dimeric density and the seven equally strong densities underneath. Interestingly, we identify strong connecting density between the alpha ring and the beta ring for all but one alpha subunit, indicating that binding of the incoming beta subunits during $\mathrm{FH}_{15 \mathrm{~S}} \mathrm{PC}$ biogenesis involves interaction with their corresponding alpha subunits. The densities of the $\mathrm{FH}_{15 \mathrm{~S}}$ PC beta ring are less well distinguishable, making it difficult to determine the exact positions of the six beta subunits and their propeptides (Supplementary Fig. 1d). The low resolution of the reconstruction (19.3 $\AA$, Supplementary Fig. 1e) and the 

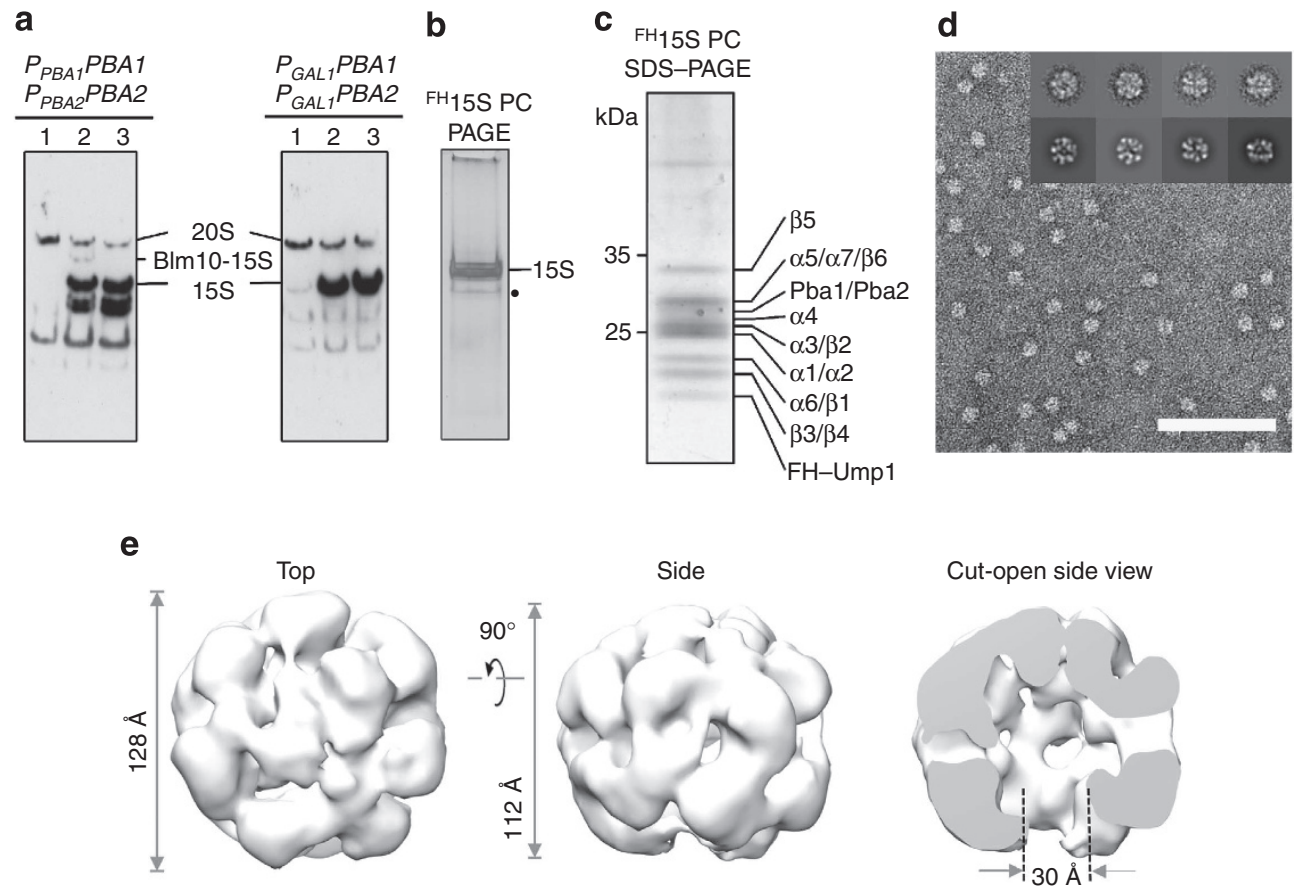

Figure 1 | Negative stain structure of the $15 S$ proteasomal precursor (PC) complex. (a) Overexpression of Pba1-Pba2 increases the amounts of $15 \mathrm{~S}$ PCs formed. Comparison of $\beta 2$-containing complexes in wild-type cells (1), or FH-UMP1 PRE4- $C$ CTE cells with (2) or without BLM10 (3) by native PAGE analysis. Left panel, wild-type expression of PBA1 and PBA2. Right panel, overexpression of PBA1 and PBA2 from $\mathrm{P}_{\text {GAL7. }}$. (b) Purified $\mathrm{FH}_{15 S}$ complex analysed by native PAGE and silver staining. (c) Same as in $\mathbf{b}$, but using $12 \%$ SDS-PAGE and Coomassie staining. Subunits of the complex were identified by peptide mass fingerprinting. (d) 15S PC micrograph with projections (inset, top row) and re-projections (inset, bottom row); scale bar, $100 \mathrm{~nm}$; (e) 15S PC 3D reconstruction based on negative stain data.

ambiguous placement of the beta subunits preclude a precise localization of Ump1 in the EM map even at lower isosurface thresholds. As the ${ }^{\mathrm{FH}} 15 \mathrm{~S}$ PC is purified via an N-terminal FH tag, this end of Ump1 appears to be easily accessible. We confirmed this notion by labelling $\mathrm{FH}-\mathrm{Ump} 1$ with 5 -nm Nanogold $\mathrm{Ni}^{2+}$ NTA particles and subsequent negative stain analysis. Nanogold binding forces the ${ }^{\mathrm{FH}} 15 \mathrm{~S}$ particles into side-view orientations (Supplementary Fig. 2a), suggesting that the $\mathrm{N}$ terminus of Ump1 is exposed at the beta ring opening because the alpha ring opening is occupied by Pba1-Pba2. A position of the Ump1 N terminus at the beta ring opening is, furthermore, consistent with the observation that an $\mathrm{N}$-terminal green-fluorescent protein (GFP)-Ump1 fusion severely inhibited cell growth and 15S PC dimerization (Supplementary Fig. 2b,c).

Ump1 cross-links to four proteasomal subunits. We complemented the EM analysis of the ${ }^{\mathrm{FH}_{1}} 15 \mathrm{~S}$ complex by CX-MS to independently verify the subunit arrangement and optimize our subunit fit into the EM density. Aside from the ${ }^{\mathrm{FH}} 15 \mathrm{~S}$ PC used for the EM analysis, we also examined a $15 \mathrm{~S}$ complex that carries a C-terminal GFP-HA tag on Ump1, but no N-terminal tag (15S GFP , Supplementary Fig. 3a). Altogether, in the N- and C-terminally tagged complexes, we identified 52 and 56 distinct intersubunit cross-links, respectively (Supplementary Tables 1-6). Out of these cross-links, 28 were found in both samples.

The majority of intersubunit cross-links were found between alpha and beta subunits, confirming a subunit arrangement that is similar to that of the mature complex (Fig. 2a, Supplementary Tables 1 and 2). Out of the 96 distinct intrasubunit cross-links in the ${ }^{\mathrm{FH}} 15 \mathrm{~S}$ sample, we detected two cross-links $(2.08 \%)$ that violated the maximum $\mathrm{C} \alpha-\mathrm{C} \alpha$ cross-link distance of $30 \AA$ (Supplementary Table 3). The rate of unfit intrasubunit cross-links in the $15 \mathrm{~S}^{\mathrm{GFP}}$ sample was at $5.21 \%$, comprising the two unfit cross-links that are also found in the ${ }^{\mathrm{FH}} 15 \mathrm{~S}$ sample plus three additional ones (Supplementary Table 4). Closer examination of all unfit cross-links reveals that each of them involves lysine residues located in loop regions of $\alpha 1$ (Lys58) or $\alpha 4$ (Lys53) or in a beta hairpin in $\beta 4$ (Lys28). Thus, we omitted these regions when fitting the proteasomal subunits as rigid bodies into the EM density of the $\mathrm{FH}_{15 \mathrm{~S}} \mathrm{PC}$. The fit was optimized manually so that subunits showed the smallest deviation from the mature complex, while simultaneously adhering to the EM map and the maximum $\mathrm{C} \alpha-\mathrm{C} \alpha$ cross-link distance of $30 \AA$ taking only intersubunit crosslinks into account that were obtained in the CX-MS analysis of $\mathrm{FH}_{15 S}$ PCs (Fig. 2b,c). Cross-links between non-neighbouring subunits were not taken into account, as they may have been formed arbitrarily (see below). Intriguingly, the CX-MS analysis shows that, apart from $\alpha 7$, all alpha subunits formed at least one cross-link to subunits of the beta ring. Thus, the lack of connecting density between one alpha subunit and the beta ring in the EM map is also reflected in the cross-linking profile of the complex. Most inter-ring cross-links appear between $\alpha 1-\alpha 4$ and $\beta 2-\beta 4$, indicating a higher structural integrity on this side of the half-barrel. This apparent feature of higher order on one side of the beta ring is also reflected in the EM map and fits previous descriptions on how the complex transitions from $13 \mathrm{~S}$ to $15 \mathrm{~S}$ precursor form (reviewed in refs 8,9,11). Together with the placement of the $\mathrm{Pba} 1-\mathrm{Pba} 2$ heterodimer in the alpha ring, it also allows us to unambiguously assign subunit densities in an otherwise pseudosymmetric complex.

In the $15 \mathrm{~S}^{\mathrm{GFP}}$ sample, no intersubunit cross-links were detected for $\beta 5$, and only one for $\beta 6$, but intrasubunit and monocross-links confirm the presence of both subunits in this complex (Supplementary Tables 4 and 5). In contrast, the ${ }^{\mathrm{FH}} 15 \mathrm{~S}$ complex displays a larger number of cross-links for both subunits, 

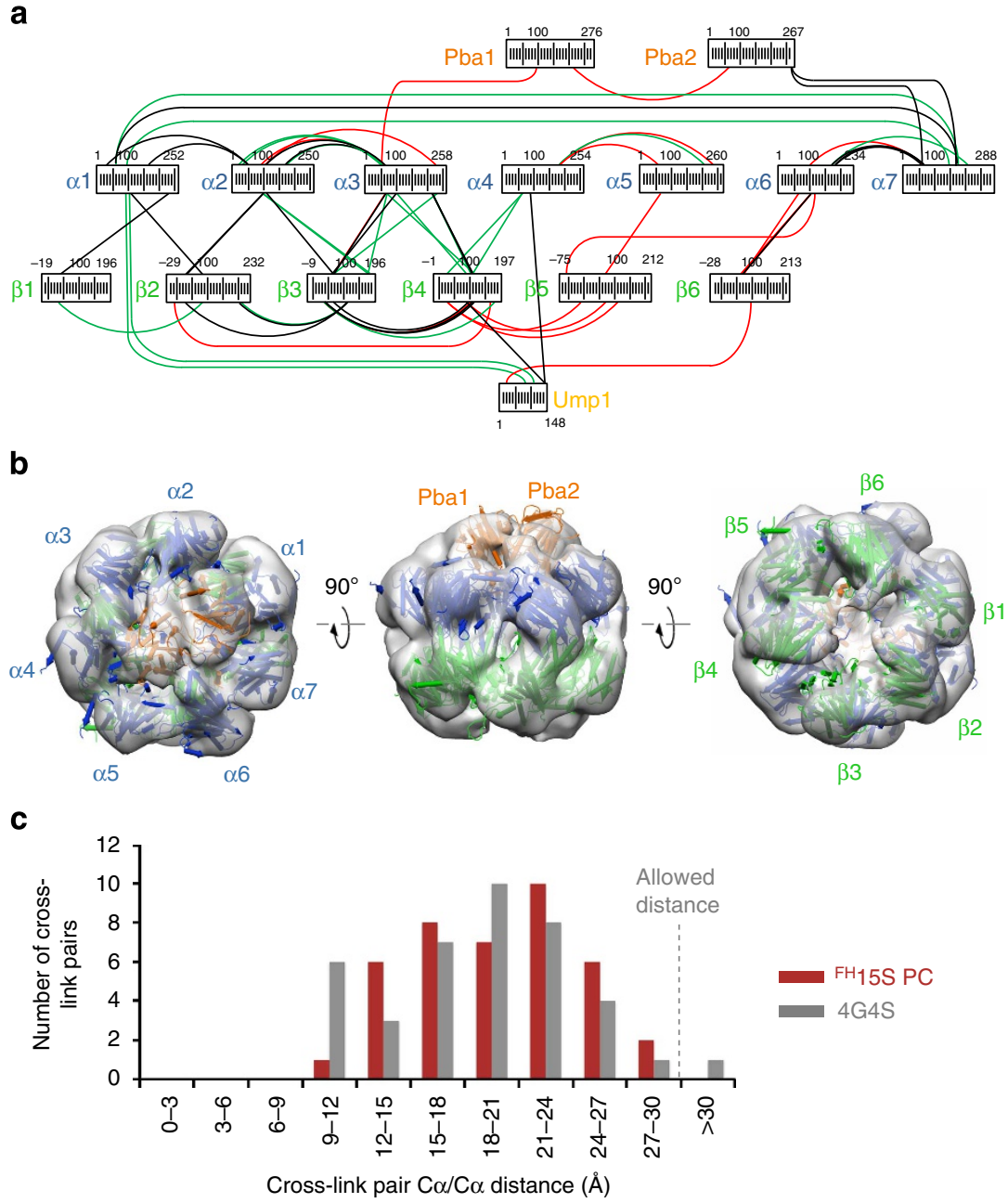

Figure 2 | Cross-linking and MS analysis of the $\mathbf{1 5 S}$ complex. (a) Overview of intersubunit cross-linking data from ${ }^{\mathrm{FH}} 15 \mathrm{~S}$ and $15 \mathrm{~S}^{\mathrm{GFP}}$ complexes investigated by MS analysis. Subunits are shown as bars with the amino-acid numbering next to them. Lines indicate the cross-links. Cross-links shown in red were found in ${ }^{\mathrm{FH}} 15 \mathrm{~S}$ complexes, those in green in $15 \mathrm{~S}^{\mathrm{GFP}}$ complexes and those in black in both complexes. GFP-related cross-links from the $15 S^{\mathrm{GFP}}$ sample and cross-links that exceed the cross-link distance of $30 \AA$ are not shown. (b) Proteasome subunits of the reconstituted Pba1-Pba2-20S complex (pdb: 4G4S) were fitted into the 15S EM map in accordance with restraints generated from cross-linking data and the EM map itself. The colour code is as follows: Pba1-Pba2 (orange), alpha1-7 (blue), beta1-6 (green) and 15S EM map (transparent grey). (c) Distances of 40 intersubunit cross-links obtained from the ${ }^{\mathrm{FH}} 15 \mathrm{~S}$ sample as shown in Supplementary Table 1 were measured on the fit into the ${ }^{\mathrm{FH}} 15 \mathrm{~S} \mathrm{EM}$ map (red) shown in Fig. $3 \mathrm{c}$ and on the reconstituted Pba1-Pba2-20S complex (grey). Distances between $\mathrm{C} \alpha$ atoms of cross-linked lysines are measured. The maximum distance covered by DSS H12/D12 and two side chains of cross-linked lysines is $\sim 30 \AA$.

indicating that the GFP moiety on the C terminus of Ump1 might alter the positioning of $\beta 5$ and $\beta 6$. Heterogeneity concerning the presence or absence of certain subunits was observed previously when the $15 S^{\mathrm{GFP}}$ complex was purified via a Strep-tag on the $\mathrm{C}$ terminus of Ump1-GFP ${ }^{33}$. Cross-links involving the GFP moiety imply a flexible position of GFP inside the chamber formed by the alpha and beta rings (Supplementary Table 6).

Of particular importance are the cross-links involving the three chaperones. The CX-MS analysis of the ${ }^{\mathrm{FH}} 15 \mathrm{~S}$ complex yielded seven intersubunit cross-links that involved Umpl, Pba1 or Pba2 (Supplementary Table 1). Four of these cross-links were also found in the $15 S^{G F P}$ sample, two of which connect the $\mathrm{C}$ terminus of $\mathrm{Pba} 2$ to $\alpha 7$, while the other two connect the $\mathrm{C}$ terminus of Ump1 (K144) to $\alpha 4$ and $\beta 4$, respectively. Thus, despite the possible re-arrangements caused by the C-terminal GFP tag in the $15 S^{\mathrm{GFP}}$ complex, Ump1 seems to be incorporated correctly. Further cross-links between Ump1 and $\alpha 1$ (for $15 S^{\mathrm{GFP}}$ ) or $\beta 6$ (for $\mathrm{FH}_{15 \mathrm{~S}}$ ) suggest that the $17-\mathrm{kDa}$ Ump1 protein stretches across the central cavity twice and is largely unfolded. Notably, cross-links between $\alpha 1$ and Ump 1 are only observed in the $15 S^{\mathrm{GFP}}$ complex. Nonetheless, we consider these cross-links valid as contacts between both proteins are also confirmed by two-hybrid data ${ }^{34}$. Altogether, three $\left({ }^{\mathrm{FH}} 15 \mathrm{~S}\right)$ and five $\left(15 \mathrm{~S}^{\mathrm{GFP}}\right)$ out of seven lysines of Ump1 formed cross-links or mono-links. All five intersubunit cross-links obtained from both samples involve Ump1 and lysines in helix 1 of alpha subunits or in helix 2 of beta subunits, all of which are located at the interface of the two rings. In the $15 \mathrm{~S}^{\mathrm{GFP}}$ PC sample, one intraprotein cross-link is formed between the $\mathrm{N}$-terminal and C-terminal segments of Ump1 in the $15 \mathrm{~S}^{\mathrm{GFP}}$ complex. Thus, our data suggest that Ump1 loops along the inside surface of the $\alpha / \beta$ ring interface, interacting with four or more proteasomal subunits.

In the final fit, all cross-links between neighbouring subunits in the ${ }^{\mathrm{FH}} 15 \mathrm{~S}$ PC cover distances of 9-30 $\AA$ between the lysine $\mathrm{C} \alpha$ atoms (Fig. 2c, Supplementary Table 1). In addition, $\sim 10 \%$ of all intersubunit cross-links obtained in the ${ }^{\mathrm{FH}} 15 \mathrm{~S}$ PC sample were found between non-neighbouring subunits, spanning distances of more than $30 \AA$ (Supplementary Table 1). All but one of these 
cross-links can be attributed to a distinct dimer between $15 \mathrm{~S}$ complexes, which was found in the ${ }^{\mathrm{FH}} 15 \mathrm{~S}$ PC sample in particular after cross-linking (Supplementary Fig. 3b). Similarly, two distinct dimers of $15 \mathrm{~S}$ complexes can explain all cross-links between nonneighbouring subunits in the $15 \mathrm{~S}^{\mathrm{GFP}}$ sample.

20S CP maturation involves re-arrangements of $\alpha$ and $\beta$ rings. In order for the $15 \mathrm{~S}$ complex to adopt the $20 \mathrm{~S}$ subunit arrangement, $\beta 7$ needs to be incorporated into the structure, the beta subunits have to move into their final positions, the alpha ring pore needs to tighten up and the $\mathrm{Pba} 1-\mathrm{Pba} 2$ chaperone has to be either expelled or degraded. A reconstituted $\mathrm{Pba1-Pba2-20S}$ crystal structure indicates that the $\mathrm{Pba}$ chaperone binds to the $\mathrm{CP}$ in its closed gate conformation, probably precluding a degradation of the chaperones ${ }^{25}$. However, the crystal structure does not reflect a proteasomal assembly intermediate and $\mathrm{Pba} 1-\mathrm{Pba} 2$ might become enclosed and/or degraded by the maturing 20S CP during its assembly in vivo. We therefore investigated late precursor intermediates purified from a strain carrying a pre1S142F (pre1-1) mutation, which severely impairs proteasomal maturation in vivo leading to 20S CPs with enclosed Ump1 and associated Pba1-Pba2 (Fig. 3a,b and Supplementary Fig. 3c) ${ }^{19,35}$. The proteasome population isolated from extracts of this strain by affinity chromatography consisted mostly of mature proteasomes, but $\sim 15 \%$ of the $20 \mathrm{~S}$ pre 1-1 proteasomes were still associated with one or two Pba1-Pba2 heterodimers (Supplementary Fig. 3d). An equal relative amount of proteasomal beta subunits was unprocessed in this population, suggesting that the Pba-containing proteasomes are late proteasomal precursors (Supplementary Fig. 1a). The 3D reconstruction of the Pba1-Pba2-20S pre1-1 complex from negative stain data at $21 \AA$ resolution reveals a very similar subunit arrangement as seen for the reconstituted Pba1-Pba2$20 S$ crystal structure, and we can dock the crystal structure as a rigid body into our EM map (Supplementary Fig. 4a).

The subunit positioning in the ${ }^{\mathrm{FH}} 15 \mathrm{~S}$ complex obtained by EM notably deviates from the arrangement in the Pba1-Pba2-20S pre1-1 complex (Fig. 3c). First, the Pba1-Pba2 densities are embedded in a cavity formed by the alpha ring, slightly pushing $\alpha 5$ and $\alpha 6$ outwards and rotating other subunits in order to create space in the central alpha ring opening. Second, the six subunits in the beta ring of the $15 \mathrm{~S}$ PC are less ordered than in the heptameric beta rings of the $20 \mathrm{~S} \mathrm{CP}$, which results in partial closure of the gap caused by the missing $\beta 7$ subunit between $\beta 1$ and $\beta 6$. The poorly organized density of the beta ring in comparison with the alpha ring suggests that incoming beta subunits bind their corresponding alpha subunits, but do not occupy their final position until $\beta 7$ incorporation or dimerization. Finally, our fit leaves some unfilled densities between $\beta 1$ and $\beta 2$ as well as $\beta 4$ and $\beta 5$, which are likely to correspond to the propeptides of the catalytic beta subunits, Ump1 or parts thereof.

$\mathrm{Pba1}-\mathrm{Pba} 2$ is expelled from the alpha ring during maturation. In comparison with the Pba1-Pba2-20S pre1-1 complex, the EM map of the ${ }^{\mathrm{FH}_{1}} 15 \mathrm{~S}$ PC reveals a strikingly closer association of Pba1-Pba2 with the rest of the complex (Fig. 4a). The alpha ring a

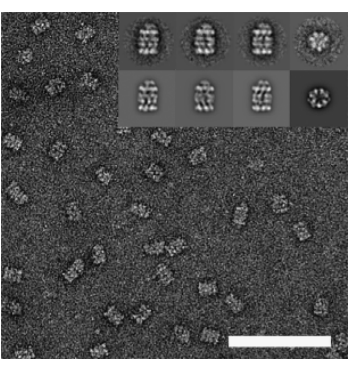

C

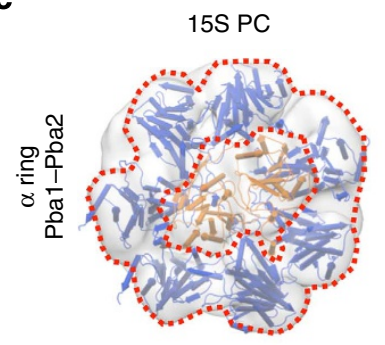

$180^{\circ}-4$

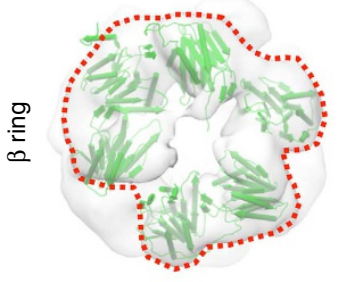

b
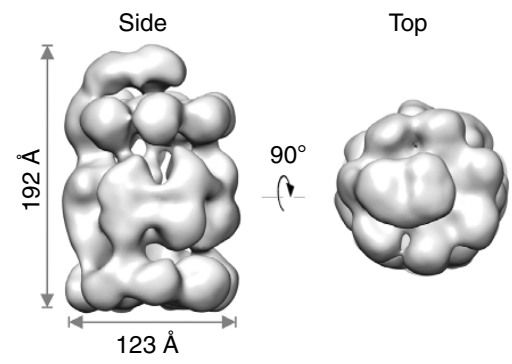

Cut-open side view

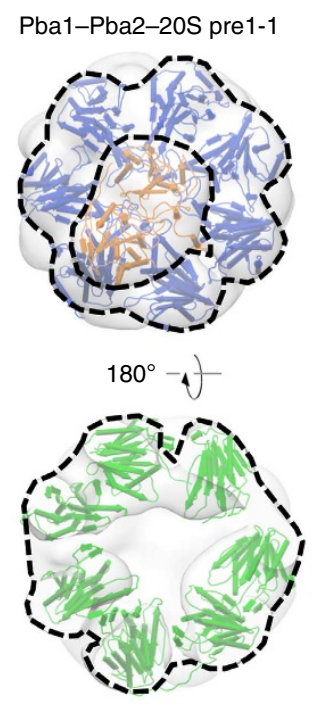

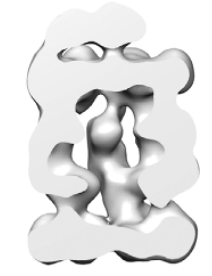

Comparison

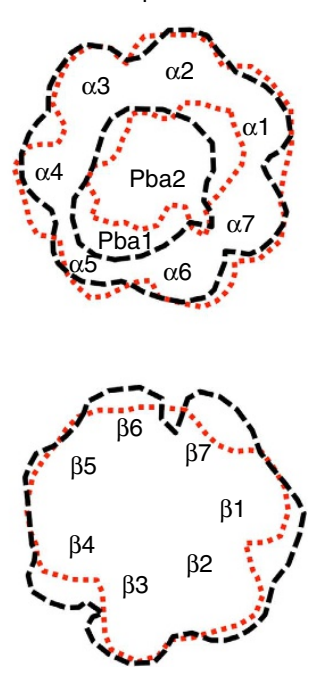

Figure 3 | Structural differences between the $\mathbf{1 5 S}$ and $\mathbf{2 0 S}$ complexes. (a) Micrograph of Pba1-Pba2-20S pre1-1 sample with projections (inset, top row) and re-projections (inset, bottom row); scale bar, $100 \mathrm{~nm}$; (b) Pba1-Pba2-20S pre1-1 3D reconstruction based on negative stain data. (c) Comparison between the subunit arrangement in the 15S precursor and the Pba1-Pba2-20S pre1-1 complex. For a better overview, the $\alpha$ ring and Pba1-Pba2 (top row) are shown separately from the $\beta$ ring (bottom row). 
opening in the ${ }^{\mathrm{FH}} 15 \mathrm{~S}$ complex is very wide with the Pba1-Pba2 heterodimer semi-embedded in a central position. In the EM map, continuous density exists between the heterodimer and all alpha subunits, except for $\alpha 5$. The strongest connections are observed near $\alpha 3$ and $\alpha 4$, as well as $\alpha 6$ and $\alpha 7$ (Fig. $4 \mathrm{~b}$ ). These interactions are not only seen in the EM map, but were also confirmed by cross-links between Pbal K91 and $\alpha 3 \mathrm{~K} 51$, as well as the Pba2 $\mathrm{C}$ terminus (K261) and $\alpha 7$ (K57 and K167; Fig. 4c) in the $\mathrm{FH}_{15 \mathrm{~S}}$ sample. $\mathrm{K} 91$ is located in a predicted loop region of $\mathrm{Pba} 1$, which was not resolved in the crystal structure; therefore, the exact architecture of this contact remains unclear. The strong interaction of the $\mathrm{Pba} 2 \mathrm{C}$ terminus with $\alpha 7$ suggests that the $\mathrm{HbYX}$ motif of $\mathrm{Pba} 2$ already engages with the $\alpha 6 / \alpha 7$ pocket during early steps of proteasome maturation. We furthermore identified one cross-link between Pba1 K213 and Pba2 K65 in the $\mathrm{FH}_{15 \mathrm{~S}}$ sample, verifying the layout of the heterodimer as seen in the crystal structure of the reconstituted complex. It is surprising that neither the EM map nor the cross-linking and MS analysis showed an interaction between Pbal and the canonical $\alpha 5 / \alpha 6$ HbYX-binding pocket. In fact, no cross-links were found between $\alpha 5$ and $\alpha 6$ either, and our fit of the crystal structures in the ${ }^{\mathrm{FH}} 15 \mathrm{~S}$ EM map indicates a rotation of $\alpha 5$, which significantly alters the layout of the binding pocket.

In comparison with the arrangement of the ${ }^{\mathrm{FH}} 15 \mathrm{~S}$ complex, the Pba1-Pba2 heterodimer is shifted out of the central cavity and moved towards $\alpha 5$ in the Pba1-Pba2-20S pre1-1 complex, giving a

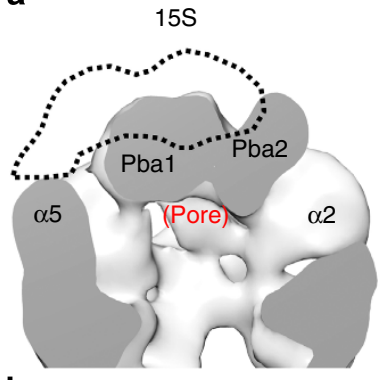

b

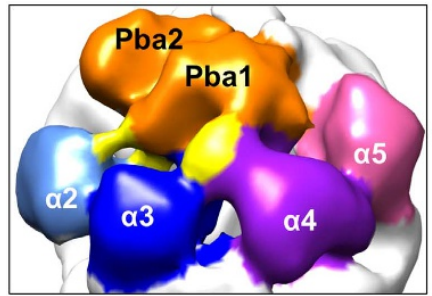

d

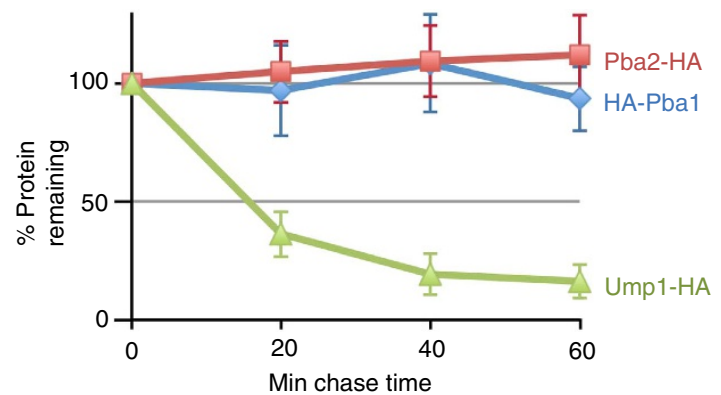

C
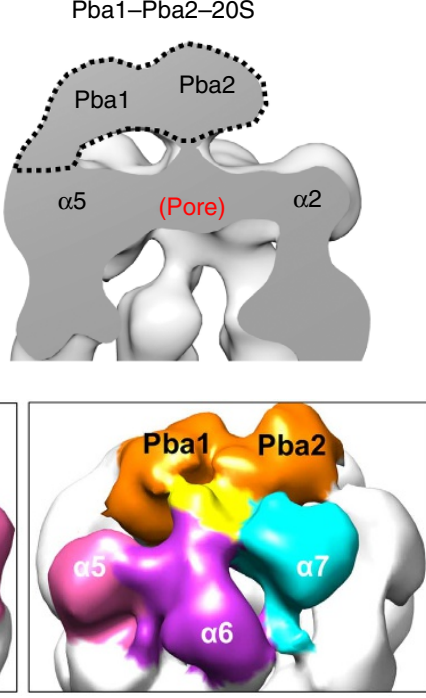
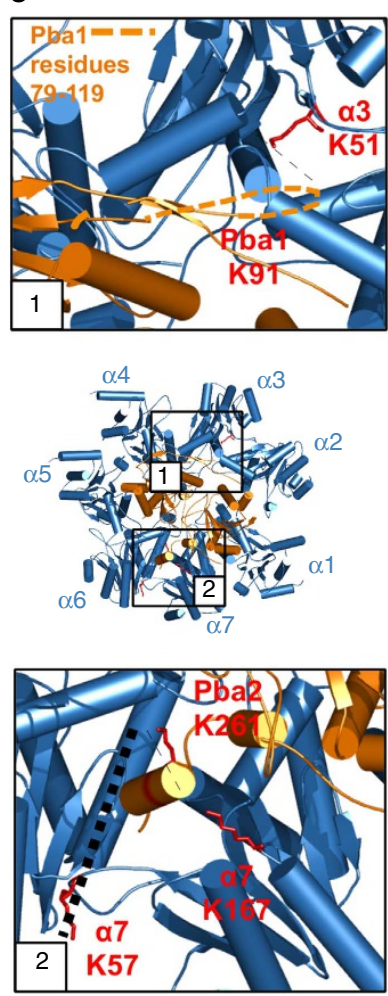
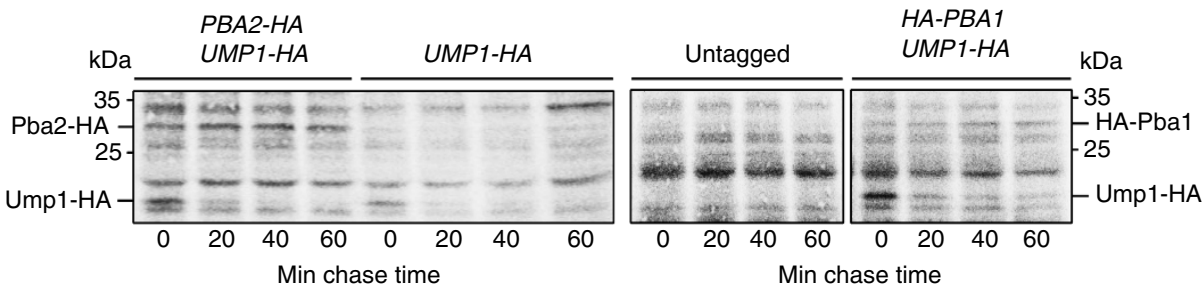

Figure 4 | Localization of Pba1-Pba2 in early and late proteasomal PCs. (a) Comparison between the positions of the Pba1-Pba2 heterodimer in the 15S precursor and Pba1-Pba2-20S pre1-1 complexes seen as cut-open side view. A black dashed line outlines the position of the heterodimer in the Pba1-Pba2$20 S$ pre1-1 complex. (b) Continuous EM density is formed between $\alpha$ subunits and the Pba1-Pba2 heterodimer in the 15S complex. EM densities corresponding to the individual subunits are coloured differently, with contact interfaces shown in yellow. (c) Cross-links found between the Pba1-Pba2 heterodimer and subunits $\alpha 3$ and $\alpha$ 7. Lysine residues are shown as red sticks and cross-links are indicated by black dashed lines. The predicted loop region of Pba1 carrying K91 is shown as an orange dashed line. (d) Pulse chase assay to monitor turnover rates of HA-Pba1, Pba2-HA, and Ump1-HA, all expressed from their native promoters and genomic loci. The middle panel shows the nonspecific background signals obtained with radiolabelled proteins from an untagged control strain. The upper part shows the results of Phosphorimager quantification of HA-Pba1, Pba2-HA, and Ump1-HA signals. Error bars were calculated using the s.d. of four independent pulse chase experiments. 
the complex an asymmetric appearance. The alpha ring pore in the structure is closed. Continuous density between Pbal and the $\alpha 5 / \alpha 6$ interface indicates an HbYX-mediated binding mode of Pbal in late PCs (Supplementary Fig. 4b). Interestingly, we also observe continuous EM density extending from Pba1$\mathrm{Pba} 2$ to $\alpha 3$, but not from $\mathrm{Pba} 2$ to $\alpha 7$, suggesting that $\mathrm{Pba} 1$ and $\mathrm{Pba} 2$ have distinct roles during different stages of proteasome maturation.

Our results indicate that yeast $\mathrm{Pba} 1-\mathrm{Pba} 2$, contrary to what had been concluded for its counterpart (PAC1-PAC2) in human cells $^{23}$, is not degraded during 20S CP biogenesis but is expelled from the structure during maturation. This conclusion suggests that, in contrast to Ump1, which is degraded by nascent proteasomes, $\mathrm{Pba} 1-\mathrm{Pba} 2$ is recycled for possible engagement in multiple rounds of 20S CP biogenesis. This notion is consistent with our observation that $\mathrm{Pba} 1-\mathrm{Pba} 2$ becomes a limiting entity in $15 \mathrm{~S}$ PC formation in the FH-Ump1 $\beta 7 \Delta \mathrm{CTE}$ background (Fig. 1a). In this strain, due to the massive accumulation of PCs and poor $20 \mathrm{~S} \mathrm{CP}$ formation, very little recycling of Pba1-Pba2 is possible, precluding engagement in multiple assembly rounds. In support of the recycling nature of $\mathrm{Pba} 1-\mathrm{Pba} 2$, we found, using the pulse chase method and chromosomally tagged strains, that $\mathrm{Pba} 1$ and $\mathrm{Pba} 2$ are metabolically stable in contrast to Ump1, which is rapidly degraded in the same cells (Fig. 4d).

\section{Discussion}

Our analysis of the 15S PC from yeast provides novel insights into the structural progression of 20S CP biogenesis as well as the roles of the assembly chaperones Ump1 and Pba1-Pba2. A trajectory map shows that Ump1 loops around the inner chamber of the 15S PC, contacting subunits at the interface of the alpha and beta rings (Fig. 5a). Contacts to alpha subunits have previously been observed for yeast Ump1 (ref. 34) and its human orthologue ${ }^{36}$. The stretched out conformation of Ump1 and the lack of defined EM density along the projected path fit its description as an intrinsically unstructured protein with little secondary structure elements $^{37,38}$. It also explains how Ump1 can be degraded by the nascent proteasome without dissolving its tertiary structure first. Earlier biochemical studies showed that Ump1 is inaccessible to polyclonal antibodies after dimerization of halfproteasomes and that apart from its $\mathrm{N}$-terminal $5-\mathrm{kDa} \mathrm{Umpl}$ is largely protected against trypsin digestion within the $15 \mathrm{~S} \mathrm{PC}^{19}$. The first 50 residues of human Ump1 were also shown to be dispensable for precursor binding, but were needed for proteasome maturation ${ }^{39}$. Our Nanogold labelling and crosslinking data indicate that a small fraction of the $\mathrm{N}$ terminus of Umpl is projecting out of the 15S PC through the beta ring opening, but the Ump1 K16- $\beta 6 \mathrm{~K} 91$ cross-link obtained in the $\mathrm{FH}_{15 \mathrm{~S}}$ sample does not suggest that large parts of Ump1 stick out of the half-proteasomes. In fact, addition of a bulky GFP moiety to the $\mathrm{N}$ terminus of yeast Ump1 blocks dimerization of the complex and inhibits cell growth (Supplementary Fig. 2c). The detected cross-link between Ump1 K16 and $\beta 6$ K91 rather suggests that the $\mathrm{N}$ terminus of Ump1 is located near the interface of $\beta 6$ and the incoming $\beta 7$ subunit; therefore, it is possibly able to sense the arrival of $\beta 7$ in the complex. The C-terminal half of $\mathrm{Ump} 1$ is required for binding to the proteasome, and cross-links to $\alpha 4$ and $\beta 4$ found in both samples suggest that this part of Ump1 associates more tightly with proteasomal subunits than the rest of the protein.

While the cross-linking data do not provide evidence of Ump1 interacting with the pro-peptide of $\beta 5$, this interaction has been suggested by genetic data from yeast ${ }^{19}$ and by biochemical data for its human orthologue hUMP1 (ref. 40). We observed crosslinks between the $\beta 5$ pro-peptide and $\alpha 6$ K115 in helix 2 in the
$\mathrm{FH}_{15 S}$ sample, which indicate close proximity between both segments. Thus, the $\beta 5$ pro-peptide seems to cross the projected path of Ump1 somewhere along the N-terminal half of Ump1, permitting a direct interaction between Ump1 and pro- $\beta 5$. The cross-link also suggests that the $\beta 5$ pro-peptide is directed towards the alpha ring rather than pointing out of the cavity, arguing against a direct role of pro- $\beta 5$ in dimerization of proteasome PCs at this stage of proteasome biogenesis, as previously discussed in ref. 16.

In the crystal structure of the reconstituted $\mathrm{Pba1}-\mathrm{Pba} 2-20 \mathrm{~S}$ complex, the heterodimer is elevated above the alpha ring, mainly contacting the $\alpha 5 / \alpha 6$ and $\alpha 6 / \alpha 7$ pockets through its $\mathrm{HbYX}$ motifs $^{25}$. In particular, the interaction between Pbal and $\alpha 6$ features conserved contacts similar to those between proteasomal activators such as Blm10 or PA26 and the $\alpha 5 / \alpha 6$ pocket. Intriguingly, our EM map and cross-linking analysis suggest a different binding mode of the heterodimer in the 15S PC. The embedded, central position of $\mathrm{Pba} 1-\mathrm{Pba} 2$, the increased pore diameter of the alpha ring and the connecting density to all alpha subunits, but $\alpha 1$ and $\alpha 5$, indicate that the heterodimer binds the $\mathrm{N}$ termini of the alpha subunits and displays a greater interaction area with the alpha ring than in late 20S PCs. Binding studies between archaeal components showed that $\mathrm{PbaA}$ requires the $\mathrm{N}$-terminal 13 amino acids of the alpha subunit for interaction with $20 \mathrm{~S} \mathrm{PCs}^{28}$. Together with $\mathrm{Pba} 3-\mathrm{Pba} 4, \mathrm{Pba} 1-\mathrm{Pba} 2$ might indeed form a structural base, on which the alpha subunits assemble into a ring structure with the correct order during early proteasome biogenesis. Furthermore, we observe cross-links and connecting EM density in the ${ }^{\mathrm{FH}_{1}} 15 \mathrm{~S}$ sample between $\mathrm{Pba} 1-\mathrm{Pba} 2$ and $\alpha 3$, the only nonessential subunit of the $20 \mathrm{~S}$ proteasome. In the reconstituted $\mathrm{Pba} 1-\mathrm{Pba} 2-20 \mathrm{~S}$ complex, no connections between the chaperones and $\alpha 1, \alpha 2$ or $\alpha 3$ are made, raising the questions whether or how Pba1-Pba2 ensures correct incorporation of these three subunits in late proteasomal precursors. Our data suggest that large loop regions in the $\mathrm{N}$-terminal region of $\mathrm{Pbal}$ contact $\alpha 3$, possibly ensuring the alpha ring integrity once $\mathrm{Pba} 3$ and $\mathrm{Pba} 4$ are dissociated.

In contrast to the mature proteasome and late PCs, the HbYXbinding pockets of the $15 \mathrm{~S}$ precursors must be distorted because the subunits of the alpha ring create a large central opening. Notably, in the $15 \mathrm{~S}$ PC, $\alpha 6$ is displaced towards $\alpha 7$, creating a greater distance between $\alpha 5$ and $\alpha 6$. This possibly prevents formation of a functional $\alpha 5 / \alpha 6$ pocket and a canonical interaction with Pba1, but allows for an intact $\alpha 6 / \alpha 7$ pocket and binding to $\mathrm{Pba} 2$. During precursor maturation, the alpha ring opening tightens and the subunits allocate to their final positions, forming the canonical HbYX-binding pockets. Tightening of the alpha and/or beta rings during maturation has previously been observed in proteasome PCs isolated from Archaeoglobus fulgidus $^{31}$ and Mycobacterium tuberculosis ${ }^{32}$, although the symmetry of those complexes, the lack of dedicated chaperones and the different nature of the beta subunit pro-peptides impede a comparison with the PCs presented in this study. In the Pba1Pba2-20S pre1-1 complex, the binding mode of the Pba1-Pba2 chaperone resembles that seen in the reconstituted Pba1-Pba2$20 \mathrm{~S}$ complex. After full maturation of the 20S, Pba1 and Pba2 are recycled for another round of proteasome maturation, indicating that their affinity to mature $20 \mathrm{~S}$ proteasomes is lower than that to the PCs. Several studies already validated this hypothesis biochemically $^{16,23,28}$, thereby also confirming the existence of changing binding modes of the $\mathrm{Pba}$ heterodimer during proteasomal biogenesis.

On the basis of our analysis of CP PCs, we suggest that the $\mathrm{Pba} 1-\mathrm{Pba} 2$ heterodimer ensures correct assembly of the alpha ring in $15 \mathrm{~S}$ complexes via its various interactions and keeps the subunits spread apart until the last beta subunit joins the PC 
a

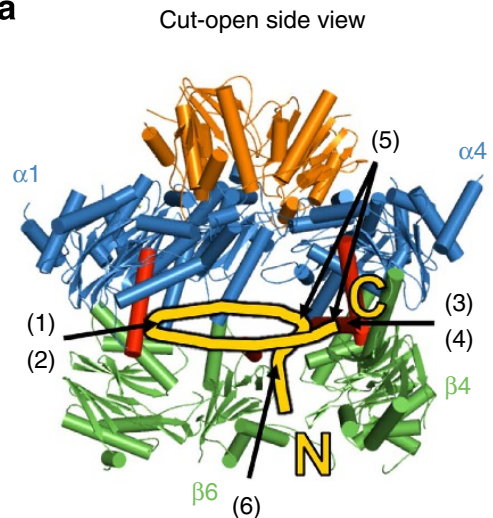

(1) $\alpha 1 \mathrm{~K} 98 / \mathrm{Ump} 1 \mathrm{~K} 83$

(2) $\alpha 1 \mathrm{~K} 107 / \mathrm{Ump} 1 \mathrm{~K} 113$
(3) $\alpha 4 \mathrm{~K} 88 / \mathrm{Ump} 1 \mathrm{~K} 144$

(4) $\beta 4 \mathrm{~K} 89 / \mathrm{Ump} 1 \mathrm{~K} 155$

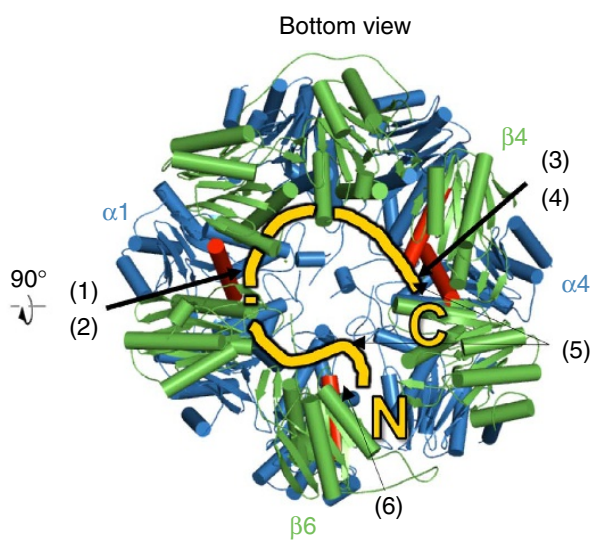

(5) Ump1 K58/Ump1 K144

(6) $\beta 6 \mathrm{~K} 91 / \mathrm{Ump} 1 \mathrm{~K} 16$

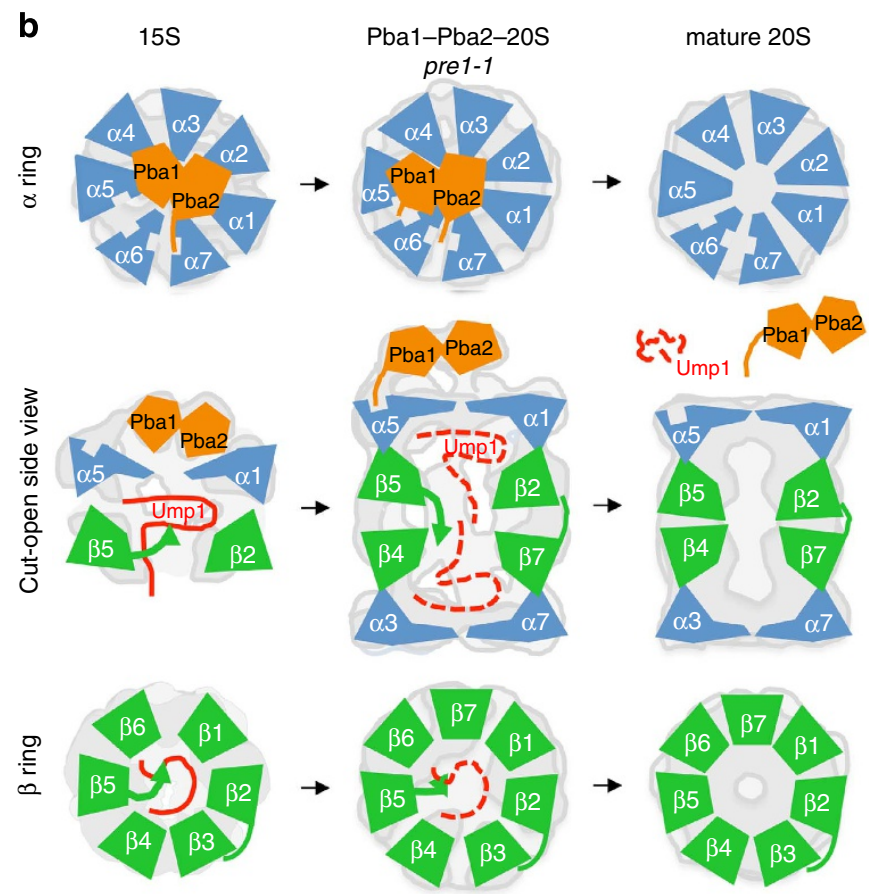

Figure 5 | Ump1 trajectory in the 15S PC and model of proteasome maturation. (a) Projected Ump1 trajectory in the $15 \mathrm{~S}$ PC shown in a cut-open side view lacking $\alpha 2 / \alpha 3 / \beta 2 / \beta 3$ (left) and bottom view lacking Pba1-Pba2 (right). Pba1-Pba2 is shown in orange, alpha subunits in blue and beta subunits in green. The projected Ump1 trajectory is shown as a yellow line. The $\mathrm{N}$ - and $\mathrm{C}$ termini are indicated. Cross-linking sites are indicated and marked by arrows. Cross-link description is colour-coded as follows: found only in $155^{\mathrm{GFP}}$ sample, green; found only in $\mathrm{FH}_{15 S}$ sample, red; found in both samples, black. $\mathrm{H} 1$ or $\mathrm{H} 2$ helices in the cross-linking subunits are shown in red. (b) Maturation pathway from $15 \mathrm{~S}$ precursor to the mature $20 \mathrm{~S}$ complex. Schematic drawings of subunits show the subunit re-arrangements during maturation. Subunits are schematically drawn in front of silhouettes generated from the $15 \mathrm{~S}$ and Pba1-Pba2-20S pre1-1EM maps as well as the low pass filtered crystal structure of the mature 20S proteasome (pdb: 1GOU, ref. 29). The subunits are colour-coded as shown in $\mathbf{a}$.

(Fig. 5b). Incorporation of $\beta 7$ into the beta ring not only establishes the layout of the beta ring, but also leads to a structural re-arrangement of the alpha ring possibly mediated by Ump1. As Ump1 is positioned ideally to sense $\beta 7$ incorporation and convey this information to other regions of the half-proteasome via its multiple interactions, we agree with previous considerations ${ }^{16}$ and suggest that Ump1 monitors the correct incorporation of subunits and prevents untimely dimerization of half-proteasomes. It possibly prevents the proteasomal subunits from prematurely locking into their final place, which would lead to expulsion of $\mathrm{Pba} 1$ and $\mathrm{Pba} 2$. Our data indicate that $\mathrm{Pba1}-\mathrm{Pba} 2$ acts as a structural chaperone that is recycled after maturation, whereas the unstructured Ump1 possibly functions as a signal transducer rather than as a structural scaffold.

\section{Methods}

Yeast media and strains. Yeast rich (YPD) and synthetic (SD) minimal media with $2 \%$ dextrose were prepared as described ${ }^{20}$.

Strain MO27 ( $P_{G A L 1}-P B A 1:: T R P 1 P_{G A L 1}-P B A 2: H I S 3$ pre4- $\triangle C T E$ FH-UMP1 blm10 $1:$ KanMX6) was derived from AM31 (ref. 18) by successively inserting the $\mathrm{P}_{G A L 1}$ promoter in front of the PBA1 and PBA2 genes using a PCR-based one-step gene transplacement strategy ${ }^{41}$. Strain MO29 (ump1 $1:: H I S 3$ PRE1-

6His::YIPlac211/URA3 pre4- $\triangle$ CTE::YIplac204/TRP1 with plasmid pMO2/CENLEU2- $P_{\text {GALS }}$ FLAG-GFP-UMP1) was derived from a JD281 spore clone (ML41$4 \mathrm{C})^{20}$ by generating the PRE1-6His allele using an integrative strategy and plasmid 
pJD315 (YIplac211-5' $\Delta$ pre1-6His- $\mathrm{T}_{C Y C 1}$ ), and subsequent transformation with pMO2. Strains MO23 (pre1-1[S142F] $]^{5}$-FH) and MO24 (PRE1-FH) were derived from the wild-type strain JD47-13C by a two-step gene transplacement strategy using plasmids pMO3 and pMO4, respectively. MO23 and MO24 were transformed with pJD657 (CEN/URA3 $P_{C U P 1}-2 x M Y C-P B A 1, P B A 2, P B A 3, P B A 4-$ $2 x H A$ ). Strains with genomically tagged UMP1 and PBA2 (MO42 and MO43) were created by consecutively transforming, respectively, strains MO23 or MO24 with the integrative plasmids pJD490 (UMP1-2HA) and pFP2 (PBA2-2HA).

Strain MK12 carrying a deletion of BLM10 and expressing Ump1 with GFP fused to the $\mathrm{C}$ terminus was created by transforming YIp5 Ump1-GFP-HA-TEVProA into BMF1 (blm104::HIS3, ref. 42).

Protein expression and purification. The ${ }^{\mathrm{FH}} 15 \mathrm{~S}$ complex was purified from a strain (MO27) grown in YP-galactose media, expressing FH-Ump1 and overexpressing $\mathrm{Pbal}$ and $\mathrm{Pba} 2$. The ${ }^{\mathrm{F}-\mathrm{GFP}} 15 \mathrm{~S}$ complex was purified from a strain (MO29) expressing ${ }^{\mathrm{F}-\mathrm{GFP}} \mathrm{Ump1}$ and Pre1 ${ }^{6 \mathrm{H}}$ grown in selective minimal media with $2 \%$ galactose. Pba1-Pba2-20S pre1-1 complexes were purified from strain $\mathrm{MO} 23$ (with pJD657) grown in selective SD media with $100 \mu \mathrm{M} \mathrm{CuSO}_{4}$. Cells derived from these cultures were pelleted and frozen in liquid nitrogen.

For preparation of the above-mentioned complexes, a tandem affinity purification protocol taking advantage of the FLAG and $6 \times$ His tags was employed. The cells were ground to powder using a Mixer Mill MM400 (Retsch) for $1 \mathrm{~min}$ at $30 \mathrm{~Hz}$. Proteins were extracted by incubating the powder with $2 \mathrm{ml} \mathrm{g}^{-1}$ wet weight FLAG buffer $(50 \mathrm{mM}$ Tris pH 7.5, $5 \mathrm{mM} \mathrm{MgCl} 2,2 \mathrm{mM}$ ATP, $150 \mathrm{mM}$ $\mathrm{NaCl}$ and $15 \%(\mathrm{v} / \mathrm{v})$ glycerol, $10 \mathrm{mM}$ imidazole with protease inhibitors (cOmplete, EDTA-free, Roche)) for $30 \mathrm{~min}$ at $4{ }^{\circ} \mathrm{C}$ with rotation. Cell debris was removed by centrifugation at $30,000 \times g$ for $30 \mathrm{~min}$ at $4{ }^{\circ} \mathrm{C}$, and the supernatant was passed through a $0.2-\mu \mathrm{m}$ filter. The filtrate was incubated with $1.5 \mathrm{ml} \mathrm{Ni}^{2+}$ sepharose resin equilibrated in FLAG buffer for $1 \mathrm{~h}$ at $4{ }^{\circ} \mathrm{C}$ with agitation. The resin was washed four times with 10 column bed volumes of FLAG buffer supplemented with $10 \mathrm{mM}$ imidazole. Bound complexes were eluted in two steps with FLAG buffer supplemented with 50 and $100 \mathrm{mM}$ imidazole. Eluted fractions were diluted 2.5 times in FLAG buffer and further incubated with anti-FLAG (M2) resin (SigmaAldrich) for $90 \mathrm{~min}$ at $4{ }^{\circ} \mathrm{C}$ with rotation. The beads were washed three times with 20 column volumes of FLAG buffer. Bound proteins were eluted with one column volume of FLAG buffer containing $0.3 \mathrm{mg} \mathrm{ml}^{-1}$ FLAG peptide (Sigma-Aldrich). Buffer was changed to either HEPES (25 mM HEPES, pH 7.5, $150 \mathrm{mM} \mathrm{NaCl}$ and $5 \%(\mathrm{v} / \mathrm{v})$ glycerol) or FLAG buffer without glycerol and ATP by using NAP-25 Sephadex G-25 columns (GE Healthcare). Eluted material was then concentrated using Vivaspin 6 columns (50,000 MWCO, Sartorius Stedim Biotech).

For preparation of the $15 S^{G F P}$ complex, MK12 yeast cells were grown in $3 \mathrm{~L}$ YPD at room temperature until an $\mathrm{OD}_{600}$ of 3.0 was reached. Cells were harvested at $3,000 \times g$, followed by washing with ice-cold $\mathrm{dH}_{2} \mathrm{O}$ and buffer A $(25 \mathrm{mM}$ HEPES, pH 7.5, $150 \mathrm{mM} \mathrm{NaCl}, 1 \mathrm{mM}$ EDTA, $5 \%$ (v/v) glycerol, $0.5 \mathrm{mM}$ dithiothreitol). Pellets were re-suspended in buffer A supplemented with $0.002 \%$ $(\mathrm{w} / \mathrm{v})$ DNase I and one cOmplete protease inhibitor tablet. Lysis was performed using a FastPrep24 (MP Biomedicals). Cell debris was removed by centrifuging at $24,000 \times g$ for $25 \mathrm{~min}$ at $4^{\circ} \mathrm{C}$. The cleared lysate was applied to IgG sepharose resin (GE Life Science) and incubated for $2 \mathrm{~h}$ at $4{ }^{\circ} \mathrm{C}$. The column material was washed three times with 20 column volumes of buffer A and PCs were eluted by incubation with TEV protease for $2 \mathrm{~h}$ at room temperature. Purified complexes were analysed by native gel and/or SDS-PAGE as well as mass spectrometry (Central Bioanalytic Unit, Center of Molecular Medicine Cologne or Gene Center, LMU, Munich).

Biochemical analysis of proteasome PCs. Gel filtration, pulse chase analysis and SDS-PAGE were performed as described before ${ }^{19}$. Native PAGE utilized Ready Gels (Tris-HCl 4-15\% gradient gels; Bio-Rad). Samples were mixed with NB4 $\times$ (240 mM Tris, pH 8.8, 80\% (v/v) glycerol and 0.04\% (w/v) bromophenol blue) and loaded onto the gel. Electrophoresis was performed in Laemmli running buffer without SDS at $16 \mathrm{~mA}$ for $\sim 1.5 \mathrm{~h}$ with refrigeration. The gels were incubated for $10 \mathrm{~min}$ in transfer buffer containing $2 \%(\mathrm{w} / \mathrm{v})$ SDS before electroblotting for $2 \mathrm{~h}$ at $0.8 \mathrm{~mA} \mathrm{~cm}^{-2}$. Membrane processing and immunodetection were performed as described $^{19}$. For pulse chase experiments, log-phase yeast cells were grown in the presence of $\left.100 \mu \mathrm{Ci}{ }^{35} \mathrm{~S}\right]$-methionine $\left(10 \mathrm{mCi} \mathrm{mmol}^{-1}\right)$ for $5 \mathrm{~min}$, followed by a chase with cold methionine for 20,40 or $60 \mathrm{~min}$. Cell extracts prepared by glass bead in lysis buffer (50 mM Na Hepes, pH 7.5, $150 \mathrm{mM} \mathrm{NaCl}, 5 \mathrm{mM}$ EDTA, $1 \%$ Triton-X100, $0.4 \%$ SDS and protease inhibitors (complete, Roche)) were subjected to immunoprecipitation with anti-HA resin (Sigma). After four washes with lysis buffer including $0.1 \%$ SDS, precipitated proteins were eluted by boiling in sample buffer, and analysed by SDS-PAGE, transferred to a nitrocellulose membrane and detected with a Typhoon FLA 7000 phosphor imager. Uncropped versions of all gel and blot scans used in the main and Supplementary Figs are presented in Supplementary Figs 5 and 6 .

Chemical cross-linking and $\mathbf{M S} .{ }^{\mathrm{FH}} 15 \mathrm{~S}$ and $15 \mathrm{~S}^{\mathrm{GFP}}$ complexes were cross-linked with 0.8 -mM disuccinimidyl suberate (DSS) H12/D12 (Creative Molecules) for $30 \mathrm{~min}$ at $30^{\circ} \mathrm{C}$ before the reaction was quenched by adding ammonium bicarbonate to a final concentration of $100 \mathrm{mM}$. To further purify cross-linked $15 \mathrm{~S}^{\mathrm{GFP}}$ complexes, they were spun through a 12 -ml gradient consisting of buffer A and 10$40 \%(\mathrm{v} / \mathrm{v})$ glycerol at $283,807 \times g$ for $16 \mathrm{~h}$. Fractions of the gradient containing $15 \mathrm{~S}^{\mathrm{GFP}}$ complexes were concentrated using Amicon Ultra centrifugal filter units $\left(100,000\right.$ MWCO, Millipore) and analysed by MS. ${ }^{\mathrm{FH}} 15 \mathrm{~S}$ complexes were analysed by MS without additional purification by a glycerol gradient. The chemical crosslinks on ${ }^{\mathrm{FH}} 15 \mathrm{~S}$ and $15 \mathrm{~S}^{\mathrm{GFP}}$ complexes were identified by MS analysis as described previously ${ }^{43}$. In short, cross-linked complexes were reduced with $5 \mathrm{mM}$ tris $(2-$ carboxyethyl)phosphine (Thermo Scientific) at $37^{\circ} \mathrm{C}$ for $15 \mathrm{~min}$ and subsequently treated with $10 \mathrm{mM}$ iodoacetamide (Sigma-Aldrich) for $30 \mathrm{~min}$ at room temperature in the dark. Digestion with lysyl enodpeptidase (Wako) at an enzymesubstrate ratio of $1-50(\mathrm{w} / \mathrm{w})$ at $37^{\circ} \mathrm{C}$ for $2 \mathrm{~h}$ was followed by a second digestion with $1 / 50(\mathrm{w} / \mathrm{w})$ trypsin (Promega) at $37^{\circ} \mathrm{C}$ overnight. Acidified peptides $(1 \%(\mathrm{v} / \mathrm{v})$ trifluoroacetic acid (TFA)) were purified by solid-phase extraction using $\mathrm{C}_{18}$ cartridges (Sep-Pak, Waters). The eluate was dried by vacuum centrifugation and reconstituted in $20 \mu \mathrm{l}$ of water/acetonitrile/TFA, 75:25:0.1. Peptides were separated on a Superdex Peptide PC $3.2 / 30$ column $(300 \times 3.2 \mathrm{~mm})$ at a flow rate of $50 \mu \mathrm{lmin}^{-1}$. Fractions of $100 \mu \mathrm{l}$ were collected and analysed by liquid chromatography coupled to tandem mass spectrometry using a hybrid LTQ Orbitrap Elite (Thermo Scientific) instrument. Fragment spectra were analysed using $\mathrm{xQuest}$ and the false discovery rate for both analyses was estimated below 3\% using $x$ Prophet $^{44}$

Electron microscopy. Negative staining of proteasome PCs was performed using 2\% $(\mathrm{w} / \mathrm{v})$ uranyl acetate. For screening of negatively stained grids, a Morgagni transmission electron microscope (FEI) equipped with an SIS Megaview 1-K chargecoupled device camera and running at $80 \mathrm{kV}$ was used. Images were collected at a nominal magnification of $\times 60,000$. For $3 \mathrm{D}$ reconstruction, micrographs were collected on a Tecnai G2 Spirit (FEI) and an Eagle CCD camera with 2,048 $\times 2,048$ pixels. The pixel size was determined to be $2.9 \AA$ at the specimen level using negatively stained $20 \mathrm{~S}$ proteasomes for calibration. The microscope utilized a lanthanum hexaboride $\left(\mathrm{LaB}_{6}\right)$ electron source set to an extraction voltage of $120 \mathrm{kV}$. For imaging, the electron dose was set to $20 \mathrm{e}^{-} \AA^{-2}$. Images were collected at defoci between -0.3 and $-1.0 \mu \mathrm{m}$. The contrast transfer functions were determined using CTFFIND3 (ref. 45) and phases were corrected for effects of the contrast transfer function in SPIDER $^{46}$. Particles were either picked manually using EMAN1 boxer ${ }^{47}$ or automatically using FindEM ${ }^{48}$. Particle stacks were subjected to sorting by XMIPP $z$ score ${ }^{49}$ and false-positive particles were excluded based on visual inspection and class editing in IMAGIC ${ }^{50}$, yielding data sets of 10,156 particles for the ${ }^{\mathrm{FH}} 15 \mathrm{~S}$ and 12,609 particles for the Pba1-Pba2-20S pre1-1 complex. After five rounds of classification based on multivariate statistical analysis followed by multireference alignment using homogenous classes as new references, eigen images of top view classifications of the $\mathrm{FH}_{15 S}$ complex and of side view classifications of the $\mathrm{Pba} 1-\mathrm{Pba} 2-20 \mathrm{~S}$ pre1-1 complex, respectively, are analysed for significant peaks indicating heterogeneity in Pbal-Pba2 binding ${ }^{51}$. Initial 3D reconstructions of $15 \mathrm{~S}$ and $\mathrm{Pba1}-\mathrm{Pba} 2-20 \mathrm{~S}$ pre1-1 complexes were created using angular reconstitution in IMAGIC. For the $15 \mathrm{~S}$ complex, initial 3D reconstruction employs $\mathrm{C} 7$ symmetry, which was relaxed to $\mathrm{C} 1$ symmetry after the first round of refinement. No symmetry was assumed during image processing of the Pba1-Pba2-20S pre1-1 data set. Euler angle assignment was refined using projection matching in SPIDER until $>90 \%$ of the Euler angles were stable. Resolutions were determined using the FSC 0.5 criterion. Three-dimensional EM reconstructions were visualized using CHIMERA ${ }^{52}$ and PYMOL (The PyMOL Molecular Graphics System, Version 1.5.0.4 Schrödinger, LLC). Contour levels of the isosurface representations were determined using chimera according to the expected molecular weight of the complexes, which are $431 \mathrm{kDa}$ for ${ }^{\mathrm{FH}_{1}} 15 \mathrm{~S}$ and $827 \mathrm{kDa}$ for Pba1-Pba2-20S pre1-1.

\section{References}

1. Crawford, L. J., Walker, B. \& Irvine, A. E. Proteasome inhibitors in cancer therapy. J. Cell Commun. Signal. 5, 101-110 (2011).

2. Ciechanover, A. \& Brundin, P. The ubiquitin proteasome system in neurodegenerative diseases: sometimes the chicken, sometimes the egg. Neuron 40, 427-446 (2003).

3. Groll, M. et al. Structure of $20 \mathrm{~S}$ proteasome from yeast at $2.4 \mathrm{~A}$ resolution. Nature 386, 463-471 (1997)

4. Lander, G. C. et al. Complete subunit architecture of the proteasome regulatory particle. Nature 482, 186-191 (2012)

5. Beck, F. et al. Near-atomic resolution structural model of the yeast $26 \mathrm{~S}$ proteasome. Proc. Natl Acad. Sci. USA 109, 14870-14875 (2012).

6. da Fonseca, P. C., He, J. \& Morris, E. P. Molecular model of the human $26 \mathrm{~S}$ proteasome. Mol. Cell 46, 54-66 (2012).

7. Smith, D. M. et al. Docking of the proteasomal ATPases' carboxyl termini in the 20 S proteasome's alpha ring opens the gate for substrate entry. Mol. Cell 27, 731-744 (2007).

8. Ramos, P. C. \& Dohmen, R. J. PACemakers of proteasome core particle assembly. Structure 16, 1296-1304 (2008).

9. Kunjappu, M. J. \& Hochstrasser, M. Assembly of the 20 S proteasome. Biochim. Biophys. Acta 1843, 2-12 (2014).

10. Saeki, Y. \& Tanaka, K. Assembly and function of the proteasome. Methods Mol. Biol. 832, 315-337 (2012). 
11. Murata, S., Yashiroda, H. \& Tanaka, K. Molecular mechanisms of proteasome assembly. Nat. Rev. Mol. Cell Biol. 10, 104-115 (2009).

12. Kish-Trier, E. \& Hill, C. P. Structural biology of the proteasome. Annu. Rev. Biophys. 42, 29-49 (2013).

13. Le Tallec, B. et al. $20 \mathrm{~S}$ proteasome assembly is orchestrated by two distinct pairs of chaperones in yeast and in mammals. Mol. Cell 27, 660-674 (2007).

14. Kusmierczyk, A. R., Kunjappu, M. J., Funakoshi, M. \& Hochstrasser, M. A multimeric assembly factor controls the formation of alternative $20 \mathrm{~S}$ proteasomes. Nat. Struct. Mol. Biol. 15, 237-244 (2008).

15. Nandi, D., Woodward, E., Ginsburg, D. B. \& Monaco, J. J. Intermediates in the formation of mouse 20 p proteasomes: implications for the assembly of precursor $\beta$ subunits. EMBO J. 16, 5363-5375 (1997).

16. Li, X., Kusmierczyk, A. R., Wong, P., Emili, A. \& Hochstrasser, M. beta-Subunit appendages promote $20 \mathrm{~S}$ proteasome assembly by overcoming an Ump1dependent checkpoint. EMBO J. 26, 2339-2349 (2007).

17. Yashiroda, H. et al. Crystal structure of a chaperone complex that contributes to the assembly of yeast 20 S proteasomes. Nat. Struct. Mol. Biol. 15, 228-236 (2008).

18. Marques, A. J., Glanemann, C., Ramos, P. C. \& Dohmen, R. J. The C-terminal extension of the beta7 subunit and activator complexes stabilize nascent $20 \mathrm{~S}$ proteasomes and promote their maturation. J. Biol. Chem. 282, 34869-34876 (2007).

19. Ramos, P. C., Hockendorff, J., Johnson, E. S., Varshavsky, A. \& Dohmen, R. J. Umplp is required for proper maturation of the $20 \mathrm{~S}$ proteasome and becomes its substrate upon completion of the assembly. Cell 92, 489-499 (1998).

20. Ramos, P. C., Marques, A. J., London, M. K. \& Dohmen, R. J. Role of C-terminal extensions of subunits beta2 and beta7 in assembly and activity of eukaryotic proteasomes. J. Biol. Chem. 279, 14323-14330 (2004).

21. Chen, P. \& Hochstrasser, M. Autocatalytic subunit processing couples active site formation in the 20S proteasome to completion of assembly. Cell 86, 961-972 (1996)

22. Groll, M. et al. The catalytic sites of $20 \mathrm{~S}$ proteasomes and their role in subunit maturation: a mutational and crystallographic study. Proc. Natl Acad. Sci. USA 96, 10976-10983 (1999).

23. Hirano, Y. et al. A heterodimeric complex that promotes the assembly of mammalian 20S proteasomes. Nature 437, 1381-1385 (2005).

24. Sasaki, K. et al. PAC1 gene knockout reveals an essential role of chaperonemediated 20S proteasome biogenesis and latent 20 S proteasomes in cellular homeostasis. Mol. Cell Biol. 30, 3864-3874 (2010).

25. Stadtmueller, B. M. et al. Structure of a proteasome Pba1-Pba2 complex: implications for proteasome assembly, activation, and biological function. J. Biol. Chem. 287, 37371-37382 (2012).

26. Dange, T. et al. Blm10 protein promotes proteasomal substrate turnover by an active gating mechanism. J. Biol. Chem. 286, 42830-42839 (2011).

27. Tian, G. et al. An asymmetric interface between the regulatory and core particles of the proteasome. Nat. Struct. Mol. Biol. 18, 1259-1267 (2011).

28. Kusmierczyk, A. R., Kunjappu, M. J., Kim, R. Y. \& Hochstrasser, M. A conserved 20S proteasome assembly factor requires a C-terminal HbYX motif for proteasomal precursor binding. Nat. Struct. Mol. Biol. 18, 622-629 (2011).

29. Groll, M. et al. A gated channel into the proteasome core particle. Nat. Struct. Biol. 7, 1062-1067 (2000).

30. Whitby, F. G. et al. Structural basis for the activation of $20 \mathrm{~S}$ proteasomes by $11 \mathrm{~S}$ regulators. Nature 408, 115-120 (2000).

31. Mullapudi, S. et al. Rearrangement of the $16 \mathrm{~S}$ precursor subunits is essential for the formation of the active 20 S proteasome. Biophys. J. 87, 4098-4105 (2004).

32. Li, D. et al. Structural basis for the assembly and gate closure mechanisms of the Mycobacterium tuberculosis 20S proteasome. EMBO J. 29, 2037-2047 (2010).

33. Lehmann, A., Niewienda, A., Jechow, K., Janek, K. \& Enenkel, C. Ecm29 fulfils quality control functions in proteasome assembly. Mol. Cell 38, 879-888 (2010)

34. Cagney, G., Uetz, P. \& Fields, S. Two-hybrid analysis of the Saccharomyces cerevisiae $26 \mathrm{~S}$ proteasome. Physiol. Genomics 7, 27-34 (2001).

35. Heinemeyer, W., Gruhler, A., Mohrle, V., Mahe, Y. \& Wolf, D. H. PRE2, highly homologous to the human major histocompatibility complex-linked RING10 gene, codes for a yeast proteasome subunit necessary for chrymotryptic activity and degradation of ubiquitinated proteins. J. Biol. Chem. 268, 5115-5120 (1993).

36. Fricke, B., Heink, S., Steffen, J., Kloetzel, P. M. \& Kruger, E. The proteasome maturation protein POMP facilitates major steps of $20 \mathrm{~S}$ proteasome formation at the endoplasmic reticulum. EMBO Rep. 8, 1170-1175 (2007).

37. Sá-Moura, B. et al. Biochemical and biophysical characterization of recombinant yeast proteasome maturation factor Ump1. Comput. Struct. Biotechnol. J. 7, e201304006 (2013).

38. Uekusa, Y. et al. Backbone $\mathrm{H}, \mathrm{C}$, and $\mathrm{N}$ assignments of yeast Umpl, an intrinsically disordered protein that functions as a proteasome assembly chaperone. Biomol. NMR Assign 8, 383-386 (2013).
39. Burri, L. et al. Identification and characterization of a mammalian protein interacting with 20 S proteasome precursors. Proc. Natl Acad. Sci. USA 97, 10348-10353 (2000).

40. Heink, S., Ludwig, D., Kloetzel, P. M. \& Kruger, E. IFN-gamma-induced immune adaptation of the proteasome system is an accelerated and transient response. Proc. Natl Acad. Sci. USA 102, 9241-9246 (2005).

41. Longtine, M. S. et al. Additional modules for versatile and economical PCR-based gene deletion and modification in Saccharomyces cerevisiae. Yeast 14, 953-961 (1998).

42. Fehlker, M., Wendler, P., Lehmann, A. \& Enenkel, C. Blm3 is part of nascent proteasomes and is involved in a late stage of nuclear proteasome assembly. EMBO Rep. 4, 959-963 (2003).

43. Herzog, F. et al. Structural probing of a protein phosphatase $2 \mathrm{~A}$ network by chemical cross-linking and mass spectrometry. Science 337, 1348-1352 (2012)

44. Walzthoeni, T. et al. False discovery rate estimation for cross-linked peptides identified by mass spectrometry. Nat. Methods 9, 901-903 (2012).

45. Mindell, J. A. \& Grigorieff, N. Accurate determination of local defocus and specimen tilt in electron microscopy. J. Struct. Biol. 142, 334-347 (2003).

46. Frank, J. et al. SPIDER and WEB: processing and visualization of images in 3D electron microscopy and related fields. J. Struct. Biol. 116, 190-199 (1996).

47. Ludtke, S. J., Baldwin, P. R. \& Chiu, W. EMAN: semiautomated software for high-resolution single-particle reconstructions. J. Struct. Biol. 128, 82-97 (1999).

48. Roseman, A. M. FindEM--a fast, efficient program for automatic selection of particles from electron micrographs. J. Struct. Biol. 145, 91-99 (2004).

49. Sorzano, C. O. et al. XMIPP: a new generation of an open-source image processing package for electron microscopy. J. Struct. Biol. 148, 194-204 (2004).

50. van Heel, M., Harauz, G., Orlova, E. V., Schmidt, R. \& Schatz, M. A new generation of the IMAGIC image processing system. J. Struct. Biol. 116, 17-24 (1996).

51. Elad, N., Clare, D. K., Saibil, H. R. \& Orlova, E. V. Detection and separation of heterogeneity in molecular complexes by statistical analysis of their twodimensional projections. J. Struct. Biol. 162, 108-120 (2008).

52. Pettersen, E. F. et al. UCSF Chimera - a visualization system for exploratory research and analysis. J. Comput. Chem. 25, 1605-1612 (2004).

\section{Acknowledgements}

We thank Cordula Enenkel for providing plasmid YIp5 Ump1-GFP-HA-TEV-ProA and strain BMF1, Stephanie Schwab for the antiserum against $\beta 5$, Filipa Pardelha for plasmid pFP2 and Thomas Fröhlich for peptide mass fingerprinting analysis. We are grateful to Michael Groll for his help in initiating this collaborative project. F.H. and colleagues are supported by the Bavarian Research Center for Molecular Biosystems, the Graduate School 1721 (DFG) and by an LMUexcellent Junior grant. M.M.N. was supported by a pre-doctoral fellowship from the Portuguese Science Foundation (FCT) and P.C.R. by a re-entry grant from the University of Cologne. P.W. and colleagues are supported by an Emmy Noether grant of the German Research Council (WE 4628/1) and the Graduate School 1721 (DFG).

\section{Author contributions}

M.M.N., R.J.D. and P.C.R. designed and performed the genetic and biochemical experiments. M.M.N. purified ${ }^{\mathrm{FH}_{1}} 15 \mathrm{~S}$ PCs and CP precursors from pre1-1 mutants for $\mathrm{EM}$ and mass spectrometric analyses. M.K. purified $15 \mathrm{~S}^{\mathrm{GFP}}$ complexes for mass spectrometric analyses and assessed all cross-links. M.H. and F.H. performed mass spectrometric analyses. The EM and 3D image analysis was designed and performed by M.K., S.K. and P.W. M.K., P.W., S.K., P.C.R. and R.J.D. contributed to data interpretation and manuscript preparation. M.K. and P.W. wrote the manuscript.

\section{Additional information}

Accession numbers: The 3D reconstructions of the 15S PC and the Pba1-Pba2-20S pre1-1 complex have been deposited in the EMBL-EBI EMDataBank under accession codes EMD-2656 and EMD-2658, respectively.

Supplementary Information accompanies this paper at http://www.nature.com/ naturecommunications

Competing financial interests: The authors declare no competing financial interests

Reprints and permission information is available online at http://npg.nature.com/ reprintsandpermissions/

How to cite this article: Kock, M. et al. Proteasome assembly from $15 \mathrm{~S}$ precursors involves major conformational changes and recycling of the Pba1-Pba2 chaperone. Nat. Commun. 6:6123 doi: 10.1038/ncomms7123 (2015). 